\title{
Visual and Instrumental Assessments of Color Differences in Automotive Coatings
}

\begin{tabular}{|c|c|}
\hline Journal: & Color Research and Application \\
\hline Manuscript ID: & COL-14-101.R3 \\
\hline Wiley - Manuscript type: & Research Article \\
\hline Date Submitted by the Author: & 31-Mar-2015 \\
\hline Complete List of Authors: & $\begin{array}{l}\text { Gómez, Omar; Universidad de Alicante, Department of Optics, } \\
\text { Pharmacology and Anatomy } \\
\text { Perales, Esther; Universidad de Alicante, Department of Optics, } \\
\text { Pharmacology and Anatomy } \\
\text { Chorro, Elísabet; University of Alicante, Dept.of Optics, Pharmacology and } \\
\text { Anatomy; } \\
\text { Burgos, Francisco Javier; Technical University of Catalonia (UPC), Center } \\
\text { for Sensors, Instruments and Systems Development (CD6) } \\
\text { Viqueira, Valentin; University of Alicante, Dept.of Optics, Pharmacology } \\
\text { and Anatomy } \\
\text { Vilaseca, Meritxell; Universitat Politecnica de Catalunya (UPC), Centre for } \\
\text { Sensors, Instruments, and Systems Development (CD6) } \\
\text { Martínez-Verdú, Francisco; University of Alicante, Optics, Pharmacology } \\
\text { and Anatomy } \\
\text { Pujol, Jaume; Universitat Politecnica de Catalunya, Centre for Sensors, } \\
\text { Instruments, and Systems Development (CD6) }\end{array}$ \\
\hline Keywords: & $\begin{array}{l}\text { Vision and color, Color measurement, Perception psychology, } \\
\text { Psychophysics, Industrial inspection }\end{array}$ \\
\hline
\end{tabular}




\title{
Visual and Instrumental Assessments of
}

\section{Color Differences in Automotive Coatings}

\author{
Omar Gómez ${ }^{*}$, Esther Perales ${ }^{1}$, Elísabet Chorro ${ }^{1}$, Francisco J. Burgos ${ }^{2}$, Valentín Viqueira ${ }^{1}$, \\ Meritxell Vilaseca ${ }^{2}$, Francisco M. Martínez-Verdú ${ }^{1}$, Jaume Pujol² \\ ${ }^{1}$ Color \& Vision Group, University of Alicante, Carretera de San Vicente del Raspeig s/n 03690, Alicante, Spain \\ ${ }^{3}$ Center for Sensors, Instruments and Systems Development (CD6), Technical University of Catalonia (UPC), \\ Rambla de Sant Nebridi, 10, 08222, Terrassa, Spain \\ *Corresponding author:omar.gomez@ua.es
}

The interest in gonio-apparent pigments (metallic, pearlescent, interference, or diffractive) has increased in the last few years, especially for applications in the automotive industry. In order to assure a proper characterization of colors with gonio-apparent pigments, commercial devices have appeared to characterize the color in different geometries, which are called multi-angle spectrophotometers. Since the gonio-apparent pigments and multi-angle instruments are relatively new, no studies exist regarding the instrumental-based procedure followed in the industry, and if the results provided are in agreement with the observer perception.

Consequently, the main objective of this study was to examine the correlation of the instrumental color differences with visual assessments. The instrumental color difference was calculated with the color difference 
formula AUDI2000 (specific for this sector) between pairs of similar samples of three types of coated panels (solid, metallic and pearlescent). The values measured by a tele-spectroradiometer in a directional lighting booth and the colorimetric values obtained by means of a multi-angle spectrophotometer BYK-mac were considered for this purpose. Additionally, a visual experiment was conducted to quantify the color difference by using the gray scale method.

The results revealed that an acceptable instrumental correlation existed despite the visual and the instrumental correlation being worse. In particular, it was checked that observers accepted a larger number of color pairs, that is, the visual color difference was smaller than the tolerance demanded by the industry (derived from AUDI2000).

\section{Introduction}

The interest in gonio-apparent pigments applied in many industries (cosmetics, inks, etc.) continues increasing day by day, especially in the automotive sector. This sector has undergone many changes and updates in the last years [1, 2], from employing only paints without visual effect to use many of them containing gonio-apparent pigments providing new and attractive visual effects by combining variable color and texture according to irradiation and viewing directions.

Goniochromatism is a change in any or all attributes of color of a specimen on change in angular illuminating-viewing conditions but without change in light source or observer [3]. This happens 
when the material that is under evaluation includes gonio-apparent pigments. Therefore, it is possible to classify materials into three types according to the pigment recipe and its colorimetric behavior: $[4,5,6]$ : solid, metallic and pearlescent or interference coatings (Figure 1, Right). The solid color only includes scattering pigments, and hence, the perceived color does not show significant changes due to the variation of the direction of lighting and/or detection. The metallic coating includes metallic pigments that provide a color shift mainly in lightness. The pearlescent coating includes in its recipe pearlescent pigments (even solid pigments) that provide a color shift mainly due to a hue and a chroma change.

Currently, in the automotive sector, color quality of automotive coatings with gonio-apparent pigments is performed by characterizing the color under different measurement geometries [5, 710]. For this, commercial devices, called multi-angle spectrophotometers, are able to characterize the color in different geometries, such as BYK-mac, or X-Rite MA98, which satisfy ASTM and DIN standards [11-14].

Although multi-angle spectrophotometers are commonly used in the automotive industry, there are few studies focused on the correlation between instrumental measurements and visual assessments by using rightly directional lighting booths [15-17]. In other words, there are not studies to check if the procedure followed in the industry, mainly based on instrumental measures of a multi-angle spectrophotometer, would match the decision that an observer would give [18]. Therefore, the conventional approach using a diffuse lighting booth and an integrating sphere spectroradiometer is not valid for gonio-apparent colors, so it is necessary to use a commercial directional lighting booth such as the gonio-vision box from Merck or the byko-spectra effect cabinet from BYK-Gardner. 
The purpose of this work is to study the correlation of the visual and instrumental assessment of the color differences between pairs of similar samples. The instrumental measurements were made with two different measurement devices: BYK-mac multi-angle spectrophotometer and PR-650 tele-spectroradiometer. In addition, a psychophysical experiment that included a directional lighting booth was designed to obtain the visual assessments in order to quantify the color difference by the comparison with a standard gray scale for color differences.

\section{Materials and Methods}

A total set of 60 pairs of different samples from solid, metallic and pearlescent pigments were selected (Fig. 1 Left). For this work knowing previously the pigment formulation of this panels was not important, though it could be interested in other future studies, because we only focused in the optical and colorimetric behavior, not structural (pigments type, size, shape, etc.). Figure 1a Left shows the $\mathrm{a}^{*} \mathrm{~b}^{*}$ and $\mathrm{L}^{*} \mathrm{C}^{*}$ chromatic diagrams with the representation of the 20 solid color pairs. The different colors indicate the different measurement geometries, and the different symbol, circle and square, the two color samples of one color pairs. Figure $1 \mathrm{~b}$ Left shows the representation at both chromatic diagrams for the metallic color pairs. And finally, Figure 1c Left shows the pearlescent color pairs. In this figure it can be seen, the color gamut associated with the selected color samples and the behavior of each kind of pigment, solid, metallic and pearlescent.

The color differences between pairs of samples were calculated by the color difference formula (1) AUDI2000 [19, 20], based on the CIELAB color space. This color difference formula was developed by and is used by AUDI, without being endorsed by any standardization body till now, with the 
main purpose to manage the color tolerances varying depending on the specific application, paint batch acceptance, color matching for add-on parts in the car body, refinish, etc.

AUDI2000 color difference formula was especially designed for materials with gonio-apparent pigments; then, it considers the changes that these materials show depending on the angle of illumination and observation (flop) as follows:

$$
\begin{aligned}
& \Delta E_{\gamma}=\sqrt{\left(\frac{\Delta L_{\gamma}}{s_{\Delta L, \gamma} g_{\Delta L}}\right)^{2}+\left(\frac{\Delta C_{\gamma}}{s_{\Delta C, \gamma} g_{\Delta C}}\right)^{2}+\left(\frac{\Delta H_{\gamma}}{s_{\Delta H, \gamma} g_{\Delta H}}\right)^{2}} \\
& s_{\Delta L_{\gamma_{i}}}=\left(\frac{\left|L_{\gamma_{i}}^{*}-L_{\gamma_{i+1}}^{*}\right|}{\gamma_{i+1}-\gamma_{i}}\right)^{2 / 3}+0.002 \cdot c_{45}^{*}+0.33 \\
& =s_{\Delta L_{\gamma i} \text {,Flop }}+s_{\Delta L_{\gamma i} \text {, Solid }}+0.33 \\
& s_{\Delta C_{\gamma_{i}}}=1.478 \cdot\left(\frac{\left|C_{\gamma_{i}}^{*}-C_{\gamma_{i+1}}^{*}\right|}{\gamma_{i+1}-\gamma_{i}}\right)+0.014 \cdot C_{45}^{*}+0.27 \\
& =s_{\Delta C_{\gamma i} \text {, Flop }}+s_{\Delta C_{\gamma i} \text {, Solid }}+0.27 \\
& s_{\Delta H_{\gamma_{i}}}=0.800 \cdot\left(\frac{\left|C_{\gamma_{i}}^{*}-C_{\gamma_{i+1}}^{*}\right|}{\gamma_{i+1}-\gamma_{i}}\right)+0.004 \cdot C_{45}^{*}+0.30
\end{aligned}
$$




$$
=s_{\Delta H_{\gamma i} \text {,Flop }}+s_{\Delta H_{\gamma i}, \text { Solid }}+0.30
$$

Where "s" are the weighting functions and "g" are the parametric factors for each term, lightness, chroma and hue, and $\gamma$ is referred to the measurement geometry, with $\gamma_{i} \in\left\{15^{\circ}, \ldots, 110^{\circ}\right\}$. To obtain a weighting for the measurement angle of $110^{\circ}$ we use half the value of the flop term from the $75^{\circ}$ angle. For the negative effect angle $-15^{\circ}$ we specified to use the weighting for the $15^{\circ}$ angle multiplied by a factor 1.2. [20].

In this study, the instrumental evaluation is carried out using two different strategies. On one hand, the color difference was calculated by using the measurement data performed by the commercial multi-angle spectrophotometer BYK-mac. This is a device that measures the samples by contact in six different geometries. This methodology is called "direct measurement", and it is the usual procedure in the automotive sector.

On the other hand, a system consisting of the tele-spectroradiometer PR-650, and the directional lighting booth, the byko-effect spectra cabinet (from BYK-Gardner) [21], were used to obtain the real spectral color stimulus and the corresponding colorimetric values of all samples without contact, never used in the automotive sector, in spite of having directional lighting booths in this sector. The main advantage of this indirect system is that it collects exactly what the observer would perceive, the PR-650 has a telecentric lens, with a measuring area of 1 deg and the distance to the spot area was about $55 \mathrm{~cm}$. For this reason, this methodology was called "(true) visual simulation". Moreover, a reference white placed at the same position as the samples was measured with the spectroradiometer to allow transformations to CIELAB color space by means of the 
absolute XYZ values (in $\mathrm{cd} / \mathrm{m}^{2}$ ), enabling us to apply absolute colorimetry into relative colorimetry, as it is usual in color appearance models [22]. The byko-effect cabinet was selected due to the fact that it allowed measuring the same geometries of illumination / observation that the BYK-mac. It is important to mention that the luminaire of the byko-effect spectra cabinet is a not a good D65 simulator, if not it is closer to a D50 simulator [23, 24]. We referred to both methodologies as direct measurement and visual simulation; however, it is important to point out that both arise from different instruments. But, obviously, the method described above can be applied to other directional lighting booths, with or not the same nominal geometries common with other multiangle spectrophotometers.

The measurement geometries used were fixed by the multi-angle spectrophotometer and the lighting booth: 45as-15, 45as15, 45as25, 45as45, 45as75, and 45as110, written following ASTM standards (Figure 2). These measurement geometries can be rewritten by following the CIE nomenclature [25] as: $45^{\circ} \mathrm{x}:-60^{\circ}, 45^{\circ} \mathrm{x}:-30^{\circ}, 45^{\circ} \mathrm{x}:-20^{\circ}, 45^{\circ} \mathrm{x}: 0^{\circ}, 45^{\circ} \mathrm{x}: 30^{\circ}$ and $45^{\circ} \mathrm{x}: 65^{\circ}$, respectively. The illumination angle was constant for all the measurement geometries.

For the visual evaluation, a psychophysical experiment was conducted by using a gray chart of the Society of Dyers and Colourists [26] for qualifying the color difference perceived in the byko-spectra effect cabinet (Figure 3 Left). The gray chart used consisted of nine neutral gray chips pairs, which are ordered in increasing color-difference. The grey scale was measured both with the BYK-mac for the experiment called "direct measurement", and with the PR650 for "visual simulation" experiment. To obtain true values for the calibrated visual assessment, that is, the visual color difference $\Delta \mathrm{V}$ which is the subjective answer provided by the observers from the visual assessments, a transformation had to be done to values that related to the color-differences 
calculated from the instrumental measurements of the grey scale. The CIELAB color-difference $\left(\Delta \mathrm{E}_{\mathrm{ab}}^{*}\right)$ was selected for this purpose as it is traditionally used for this transformation [27]. The grey pairs were measured at each angle, following the same methodology applied in the previous work [19], and a fourth polynomial fitting was found to be the best to adjust the values in the scale colordifferences obtained through instrumental measurements for each of these observation angles (Figure 3 Right). Thanks to this adjustment, the visual color difference $(\Delta \mathrm{V})$ for a given angle can be interpolated and directly related to $\Delta \mathrm{E}^{*}$ ab. For this experiment, 10 observers were included (5 men and 5 women) with normal color vision. In each 30-min session, the observer performed 120 evaluations, having a total of 1080 evaluations done in 9 sessions (3 repetitions in each session). The color pairs and the different measurement geometries were randomly presented to the observers. Before each session, the observer spent 3 minutes to adapt to the illuminance level and cabinet environment. The observer's task was to quantify the color difference in the test pair by comparing with the color differences shown in the several gray pairs (Figure 3 Left).

The STRESS index (standardized residual sum of squares) was used to evaluate the performance of the color difference formula with respect to the visual assessments obtained [28, 29]. As a consequence, the lower the STRESS values, the better the correlation. In addition, the STRESS index was used not only to estimate the color difference performance but also to know how reliable the results were by determining the intra- and inter- observer variability [30].

The intra-observer variability refers to the differences between the results obtained when the same observer reported more than once on the same issue. It was measured by the STRESS index, calculated from 3 repetitions for each observer at the same experiment. The inter-observer variability refers to the differences between the results produced by different observers. It was also 
calculated by means of the STRESS index, considering the total average of the 10 observers and the mean value of 3 replicates of each observer.

\section{Results}

The psychophysical assessments and the instrumental data allowed determining the instrumental and visual correlation regarding the experiment executed.

\section{Observer Variability}

The average intra-observer variability obtained in this experiment was 23.65 STRESS units and the average inter-observer variability was 28.25 STRESS units. Although there were two observers who showed high intra-variability, the calculated values were good compared with previous studies $[19,31]$.

\section{Instrumental Correlation}

Before analyzing the visual and instrumental correlation, an instrumental correlation between the two measurement methodologies (direct measurement and visual simulation) was done. The AUDI2000 color difference $\left(\Delta \mathrm{E}_{\mathrm{AUDI}}{ }_{000}\right)$ was calculated by considering the measurements from the BYK-mac multi-angle spectrophotometer, fixating the D65 illuminant, and the measurement from the PR-650 tele-spectroradiometer from the true spectral stimuli, that is, the combination of the true lamp into the booth and the spectral reflectance of the panel for this measurement geometry. Next, a correlation of these instrumental data (BYK-mac and PR-650) was performed by using a graphical analysis in which the values of $\Delta \mathrm{E}_{\mathrm{AUDI}} 2000$ obtained in the different conditions were compared. 
Ideally, a linear correlation of slope close to 1 in $\Delta \mathrm{E}_{\mathrm{AUDI}}{ }_{2000}$ values was expected. These values were generated with both measuring instruments for each of the measurement geometries. In these conditions a pair of panels would exhibit similar color differences by using the BYK-mac multiangle spectrophotometer $\left(\Delta \mathrm{E}_{\mathrm{BYK}-\mathrm{mac}}\right)$ and by using the PR-650 tele-spectroradiometer and the cabinet $\left(\Delta \mathrm{E}_{\mathrm{PR} 650}\right)$ even though they were analyzed with the BYK-mac and the telespectroradiometer PR-650 plus the lightning booth, respectively. In addition, the correlation would be linear in spite the fact that the method that involves the tele-spectroradimeter and the lightning booth contains higher variability in the position/direction of the instrument, orientation of panels, and the spectral quality of the daylight D65 simulation installed into the lighting booth.

Plots in Figure 4 show the correlations in terms of the color differences $\left(\Delta \mathrm{E}_{\mathrm{PR}-650 \text { and }} \Delta \mathrm{E}_{\mathrm{BYC}-\mathrm{mac}}\right)$ for the 60 color pairs classified by measurement geometry. There was a good correlation between both instruments and methods, although it was not perfect. The differences found might be due to several factors. Firstly, the fact that the light source (fluorescent lamp) was not the same. Firstly, although using the BYK-mac the reference illuminant is the D65, the light source of the byko lighting booth, both belonging to the same company, is closer to the D50 illuminant [30, 31]. Secondly, the illumination / observation conditions may be slightly altered between various measurements, and this is especially relevant in goniochromatic samples where color strongly depends on the measurement configuration.

It is worth to point out that the correlation between both methodologies was poorer for the geometry 45 as- 15 . Whilst the $45 \mathrm{as}^{-15}$ and $45 \mathrm{as} 15$ geometries were equally close to the specular direction, the geometry of 45 as- 15 was experimentally the most conflicting, since in this direction the intensity of the reflected light was higher than in other more distant specular geometries. In 
general, a great spreading was found for the geometries 45as-15 and 45as110. Moreover, some color pairs did not follow a linear behavior as expected. As these measurement geometries are the extreme ones in both instrumental configurations, this could the reason why a worse correlation is found, specifically due to some opto-mechanical design problems for applying telespectroradiometry on a tilted scene. However, for 45as25, $45 a s 45$ and $45 a s 75$ geometries which progressively move away from the specular peak, the intensity of the reflected light decreased the goniochromatic effects, there was less spreading in characterizing the samples and the color difference results matched for both procedures.

\section{Visual and Instrumental Correlation}

The next step was the evaluation of the visual and instrumental correlation. As it was mentioned before, the correlation was evaluated by the STRESS index. Therefore, this index was calculated for both instrumental color differences (BYK-mac and PR-650) by considering the visual differences obtained from the psychophysical experiment. In Figure 5, a histogram is shown for the STRESS values calculated for both color differences and measurement geometry. As it can be seen, the results were very similar for both color differences. The STRESS average for all geometries was of 39.78 and 40.87 units for BYK-mac and PR-650 data, respectively. This result was already expected as the correlation between both instruments was acceptable as previously shown.

\footnotetext{
A set of color-pairs was identified having high deviations between the visual assessment and instrumental measurement, even for both methodologies. These are types of samples that would cause problems in the industry. Therefore the multi-angle spectrophotometer would not accept the color pair as a good color matching, but an observer would.
} 
Furthermore, color-pairs that generated conflicts, i.e. the response of the instrument was different from the observer's decision, were identified and selected. Therefore, a new analysis was done in order to show for each color-pair, the correlation of both processes, instrumental and visual evaluation, in each measurement geometry. This analysis consisted of doing a representation (Figure 6) by denoting with zones the criteria established by the AUDI color tolerances. In this way, it was easy to visualize if the samples presented acceptable color differences or not.

Based on the AUDI tolerance criteria, all samples with $\Delta \mathrm{E}>1.7$ units would be placed within the red zone and would be considered as different; samples with color differences between 1.4 and 1.7 would be at the yellow zone, where samples should be studied with other visual tests to establish if they pass or not; finally, the green area would contain samples with $\Delta \mathrm{E}<1.4$, which could be considered almost indistinguishable.

Once the most troubled samples were identified and analyzed, 3 pairs of characteristic samples were selected to be shown in Figure 6. In this case, measurement data from the BYK-mac was used as it is the most common instrument used in the automotive industry.

Figure 6a shows the color differences for all the measurement geometries for a solid sample. As it can be noticed, there was a good correlation between visual and instrumental assessments, which means that for both methodologies the color difference of the color pair was indistinguishable (green region). This situation should be the real one, where the information provided by the instrumental analysis and visual evaluation would be equivalent.

Figures $6 \mathrm{~b}$ and $6 \mathrm{c}$ show an opposite situation. These examples correspond to pearlescent and metallic samples, respectively. In the pearlescent sample, the color-pair was accepted by the BYKmac (for all the measurement geometries) but rejected by the average observer. On the contrary, 
for the metallic sample the average observer accepted the color-pair as a good color matching or approval, whereas the color difference calculated from the BYK-mac measurements was big enough to consider the color-pair as not good or fail. Therefore, it would be interesting for car makers and coating providers to know what type of automotive colors or recipes (by hues, flops, etc.) are prone to be associated with the cases shown in these figures.

\begin{abstract}
It is important to mention that with the PR-650 data, results were similar to those obtained using the BYK-mac, since in the majority of samples, pairs were accepted by observers, but rejected by the instrument, as for the BYK-mac.
\end{abstract}

\title{
4. Conclusions
}

The correlation between visual and instrumental evaluation was performed with a set of automotive samples. Two instrumental evaluations were conducted, a direct measurement with the BYK-mac multi-angle spectrophotometer, and a visual simulation with the PR-650 telespectroradiometer and the byko-effect lighting cabinet, with the same measurement geometries setup than in the BYK-mac. The visual evaluation was conducted by a conventional psychophysical experiment where observers quantified the color difference in color pairs by using the gray scale method. The observer variability showed that the observer responses were consistent enough.

Firstly, a comparison between both instrumental evaluations was done. The results exhibited an acceptable correspondence between both methodologies. Nevertheless, a slightly measurement mismatch that could be due to the illuminant - light source differences or different geometries configurations was found. 
Secondly, the study of the visual and instrumental correlation was carried out. The performance of the AUDI2000 color difference with the visual assessments done by observers was evaluated by the STRESS index. The results revealed that it would be possible to obtain a better performance by improving the color difference formula since the observer variability was lower than the performance of the color difference. A detailed analysis was done for each color pair. A number of color pairs were identified to have instrumentally color differences far away from visual color differences. It was determined that the general tendency was that observers gave lower color difference values than those provided by the devices. This implies that observers accepted a larger number of valid color pairs compared with those accepted by the devices, despite being "experts" in color, although not color automotive engineers. Therefore, it could be concluded that there was not a perfect correlation between instrumental and visual evaluation and that it would be interesting to analyze which parameters can influence, and how, in this correlation failure related with commercial color devices typically used in the automotive sector. One approach would be to improve the AUDI2000 color difference formula or to propose a new one in order to obtain a better performance. Taking into account that the observer variability was lower than the performance of the color difference, it would be possible to improve the AUDI2000 color difference formula to find a better correlation with visual evaluations. Other strategies, even combining with the first approach, would be to use a better D65 simulator, a more powerful lamp for a higher level of illumination, better lighting uniformity, or even use a specified gray card with smaller steps to compare directional level colors. This experiment should also be done with professional colorists of the automotive industry and with normal observers (with no experience in colorimetry), to see whether the level of experience affects $\Delta \mathrm{V}$. Therefore, this justifies that it is necessary to apply both methodologies, visual and instrumental evaluation, to avoid this kind of discrepancies. Otherwise, 
working hardly in the improvement of directional lighting booths, using better D65 simulators and high illuminance levels, either based on current fluorescent lamps or new solid-state lighting sources as LEDs, OLEDs, etc.

In conclusion, the new main contributions of this paper is the use of tele-spectroradiometry for testing the visual and instrumental correlation in automotive coatings at the realistic way, showing that this instrumental technique for measuring the true spectral stimuli is necessary to understand and manage doubtful color pairs for the final color matching/approval. And it can be applied to new experiments and studies crossing structural (formulation, pigment size, etc.) and colorimetric data.

\section{Acknowledgments}

The authors are grateful to EMRP for funding the project "Multidimensional Reflectometry for Industry". The EMRP is jointly funded by the EMRP participating countries within EURAMET and the European Union. We would like to thank the Ministry of Economy and Competitiveness for the coordinated project "New developments in visual optics, vision and color technology" (DPI2011-30090-C02). Francisco Javier Burgos would also like to thank the Autonomous Government of Catalonia for his pre-doctoral fellowship grant and Omar Gómez to the Ministry of Economy and Competitiveness for his pre-doctoral fellowship (FPI BES-2012-053080). 


\section{References}

1. Brock, T., Groteklaes, M., Mischke, P., European Coatings Handbook. Hannover: Vincentz Network. 2010

2. Streitberger, H.J., Dössel, K.F., Automotive Paints and Coatings, 2nd ed. Wienheim: Wiley-VCH. 2008

3. ASTM E284-13b. Standard Terminology of Appearance. Conshohocken: American Society for Testing Materials.

4. Maile, F.J., Pfaff, G., Reynders, P., "Effect pigments - past, present and future.” Prog. Org. Coat., 54, 150-163. 2005

5. Klein, G.A., Industrial Color Physics. New York: Springer. 2010

6. Pfaff, G., Special Effect Pigments. 2nd ed. Hannover: Vincentz Network. 2008

7. Chorro, E., Martínez-Verdú, F., campos, J., Pons, A., "Colorimetric and spectral evaluation of the optical anisotropy of metallic and pearlescent samples.” Journal of Modern Optics, 56, 13, 1457-1465. 2009

8. Schanda, J., Colorimetry: Understanding the CIE System. New York: John Wiley \& Sons. 2007

9. Baba, G., Suzuki., "Gonio-spectrophotometric analysis of white and chromatic reference materials." Anal. Chim. Act., 380, 173-182. 1999

10. E. J. J. Kirchner and J. Ravi, "Setting tolerances on color and texture for automotive coatings," Color Res. Appl., 39(1), 88-98 (2012).

11. DIN-6175-2. Tolerances for Automotive Paint. Part 2: Goniochromatic Paints. Berlin, Germany: Deutsches Institut für Normung; 2001.

12. ASTM E2194-12. Standard Practice for Multiangle Color Measurement of Metal Flake Pigmented Materials. Conshohocken: American Society for Testing Materials.

13. ASTM E2539-12. Standard Practice for Multiangle Color Measurement of Interference Pigments. Conshohocken: American Society for Testing Materials.

14. Perales E, Chorro E, Viqueira V, Martınez-Verdu FM., "Reproducibility comparison among multiangle spectrophotometers." Color Res Appl 2013;38:160-167.

15. M. Huang, G. Chen, H. Liu, G. Cui, M.R. Luo, N. Liao, M. Melgosa, Y. Zhang, C. Zheng, "A comparison of different psychophysical methods for color-difference evaluation,” in Proceedings AIC Colour 2013 (Newcastle, UK, 2013),

16. O. Gómez, F. J. Burgos, E. Perales, E. Chorro, V. Viqueira, F. M. Martínez-Verdú, and J. Pujol, "Preliminary comparative performance of the AUDI2000 and CIEDE2000 color difference formulas by visual assessments in directional lighting booth," in Proceedings AIC Colour 2013 (Newcastle, UK, 2013), 4, pp. 1545-1548.

17. F. J. Burgos, E. Perales, O. Gómez, E. Chorro, V. Viqueira, F. M. Martínez-Verdú, and J. Pujol, "Instrumental and visual correlation between a multiangle spectrophotometer and a directional lighting booth," in Proceedings AIC Colour 2013 (Newcastle, UK, 2013), 4, pp. 1373-1376.

18 W. V. Longley, “Automotive color certification,” Color Res. Appl. 20(1), 50-54 (1995).

19. Melgosa, M, Martínez-García J., Gómez-Robledo L., Perales E., Martínez-Verdú FM., Dauser T., "Measuring color differences in automotive samples with lightness flop: a test of the AUDI2000 color-difference formula." Opt. Express 10; $22(3): 3458-67.2014$

20. Dauser, T., Audi color tolerance formulas (Private communication). 2012

21. https://www.youtube.com/watch?v=7j6oBkDKnjE Experience the byko-spectra effect.

22. Fairchild, Mark D. Color Appearance Models, Published Online: 18 JUN 2013. DOI: 10.1002/9781118653128.ch20. 
23. Perales, E., Chorro, E., Werner, C., Viqueira, V., Gómez O., Martínez-Verdú, F.M., "Influence of spectral power distribution of light sources on the color appearance of goniochromatic colours." CIE Centenary Conference, Paris. 2013

24. Martínez-Verdú, F., Perales, E., Viqueira, V., Chorro, E., Burgos, F.J., Pujol, J., "Comparison of colorimetric features of some current lighting booths for obtaining a right visual and instrumental correlation for gonio-apparent coatings and plastics." Proceedings of CIE 2012 "Lighting Quality and Energy Efficiency", 692-705. 2012

25. Commission Internationale de l'Éclairage (CIE), Colorimetry, 3rd Edition, CIE Publication 15:2004 (CIE Central Bureau, Vienna, 2004).

26. http://WWw.sdcenterprises.co.uk/products/sdc-assessment-aids/grey-scale/ See also ISO 105-A02:1993, "Test for colour fastness - Part A02: Gray scale for assessing change in colour," International Organization for Standardization (Geneva, Switzerland).

27. Guan, S. S. \& Luo, M. R., "Investigation of parametric effects using small colour differences." Color Research and Application 24, 331-343. 1999

28. García, P. A., Huertas, R., Melgosa, M., \& Cui, G., "Measurement of the relationship between perceived and computed color differences." Journal of the Optical Society of America A, 24(7), 1823-1829. 2007

29. Kirchner, E. \& Dekker, N., "Performance measures of color-difference equations: correlation coefficient versus standardized residual sum of squares." Journal of the Optical Society of America A, 28(9), 1841-1848. 2011

30. Melgosa, M., García, P. A., Gómez-Robledo, L., Shamey, R., Hinks, D., Cui, G., \& Luo, M. R., "Notes on the application of the standardized residual sum of squares index for the assessment of intra- and inter-observer variability in color difference experiments." Journal of the Optical Society of America. A, 28(5), 949-53. 2011

31. Huang, M., Liu, H., Guihua C., Ronnier Luo, M., Melgosa, M., "Evaluation of threshold color differences using printed samples." Journal of the Optical Society of America A, 29, 6, 883-891. 2012 
Figure 1 Left: Color appearance of the 60 assessed color pairs, Right: Colorimetric behavior according to the pigment recipe.

Figure 2: Schematic representation of the illumination / measurement geometries used with the BYK-mac device for color characterization.

Figure 3 Left: Which gray pair is closer to the color difference seen in the color pair?. Right: CIELAB color differences for each one of the nine color pairs in the SDC gray scale. Fourth Degree Polynomial Fit, $R^{2}(-15)=0.9849, R^{2}(15)=0.9938, R^{2}(25)=0.9975, R^{2}(45)=0.9998$, $\mathrm{R}^{2}(75)=1, \mathrm{R}^{2}(110)=1$

Figure 4: Instrumental correlation obtained from the color differences of the instruments for the geometries 45as-15, 45as15, 45as25, 45as45, 45as75, 45as110. Different colors are used to identify the sample type (blue: solid, red: metallic and green: pearlescent pigment).

Figure 5: STRESS values calculated for both instrumental color differences.

Figure 6: Color differences for all the measurement geometries for a) solid sample, b) pearlescent sample and c) metallic sample following the criterion Passes / Not Passed. The green region means almost indistinguishable color differences, the red zone means rejected color pairs and the yellow zone means critical color differences, samples need to be visually assessed by expert colorists. 

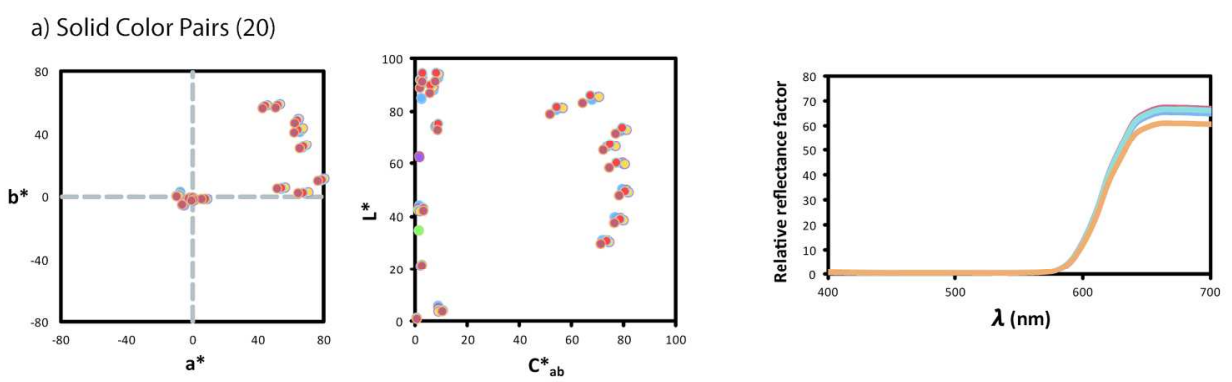

b) Metallic Color Pairs (20)
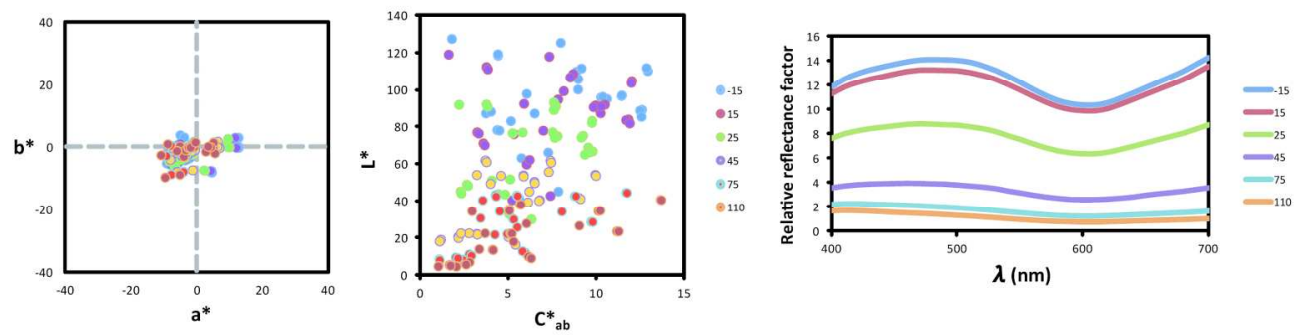

c) Pearlescent Color Pairs (20)
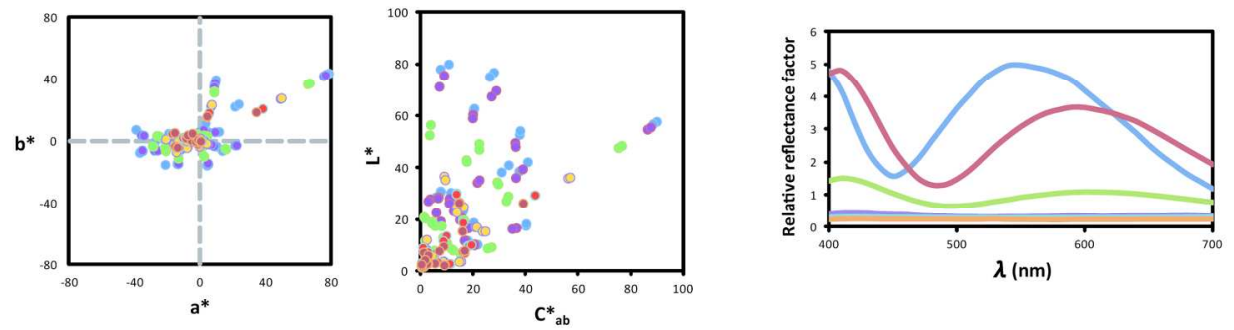

Figure 1 Left: Color appearance of the 60 assessed color pairs, Right: Colorimetric behavior according to the pigment recipe.

$176 \times 155 \mathrm{~mm}(300 \times 300$ DPI $)$ 
Figure 2: Schematic representation of the illumination / measurement geometries used with the BYK-mac device for color characterization. $88 \times 55 \mathrm{~mm}(300 \times 300 \mathrm{DPI})$ 

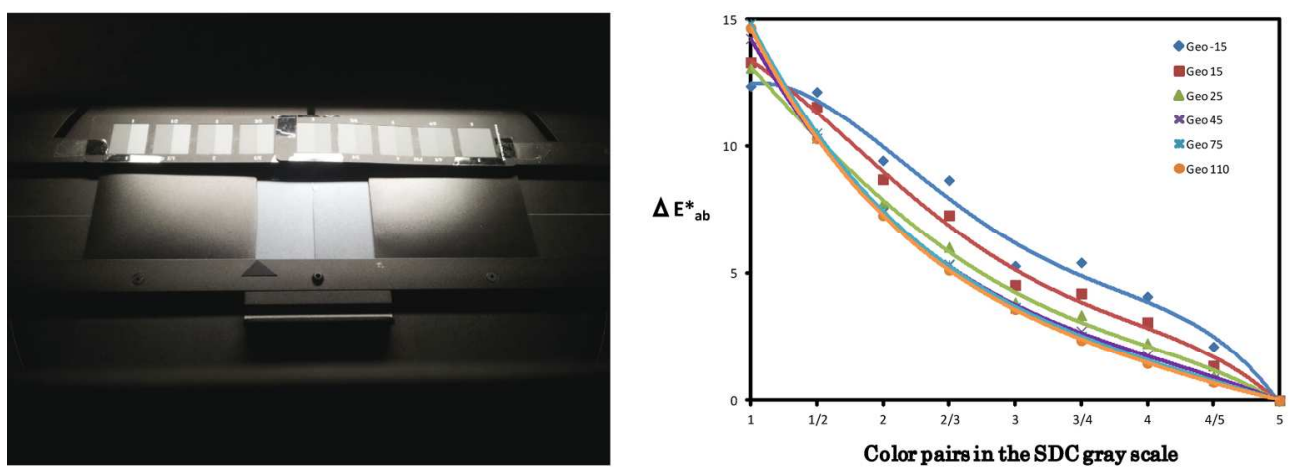

Figure 3 Left: Which gray pair is closer to the color difference seen in the color pair?. Right: CIELAB color differences for each one of the nine color pairs in the SDC gray scale. Fourth Degree Polynomial Fit, $R^{2}(-15)$ $=0.9849, R^{2}(15)=0.9938, R^{2}(25)=0.9975, R^{2}(45)=0.9998, R^{2}(75)=1, R^{2}(110)=1$. $177 \times 64 \mathrm{~mm}(300 \times 300 \mathrm{DPI})$ 

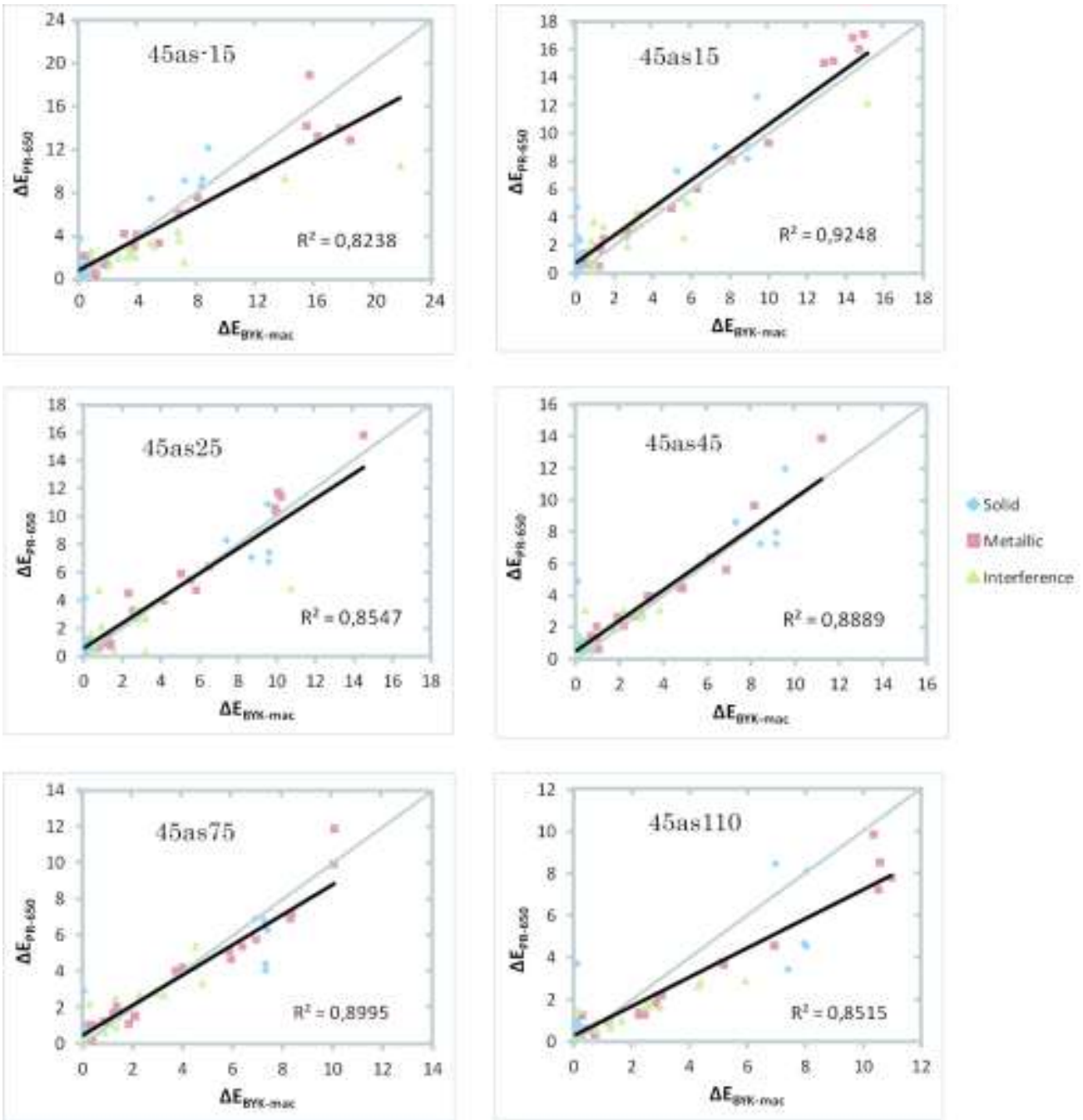

Figure 4: Instrumental correlation obtained from the color differences of the instruments for the geometries 45as-15, 45as15, 45as25, 45as45, 45as75, 45as110. Different colors are used to identify the sample type (blue: solid, red: metallic and green: pearlescent pigment). $122 \times 126 \mathrm{~mm}(300 \times 300 \mathrm{DPI})$ 


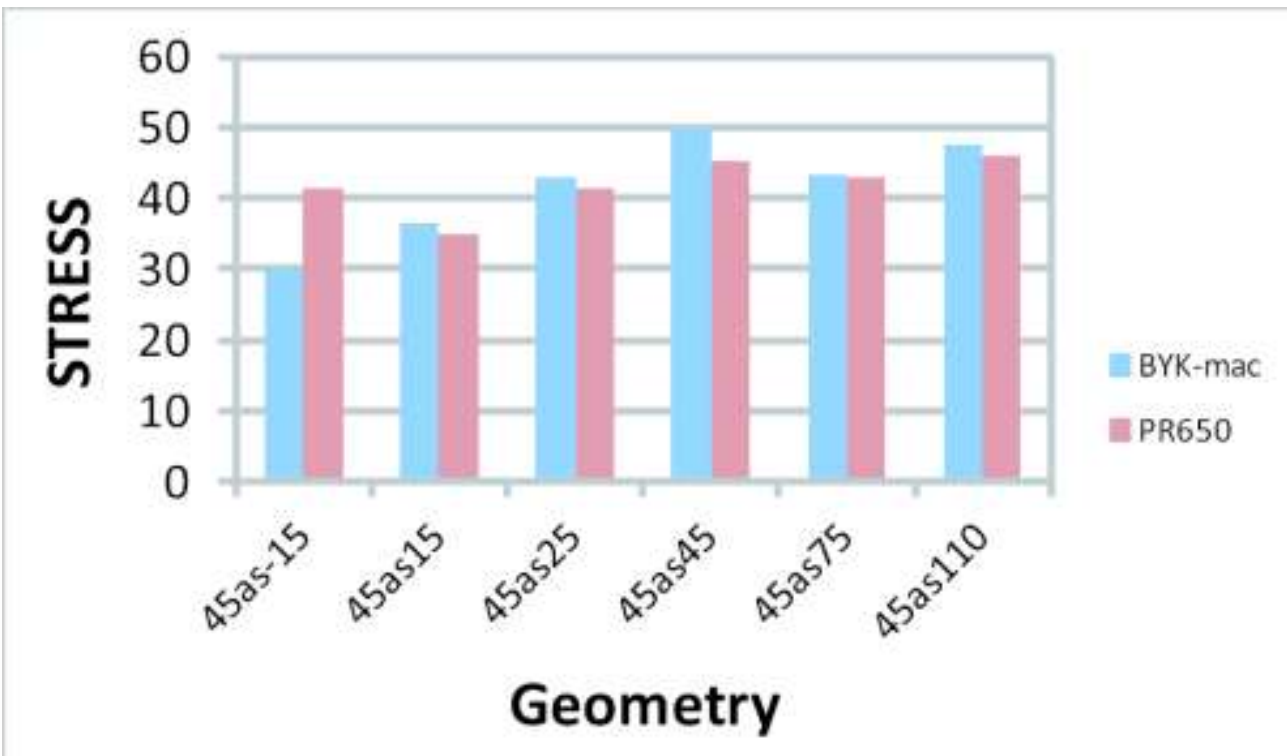

Figure 5: STRESS values calculated for both instrumental color differences. $88 \times 53 \mathrm{~mm}(300 \times 300$ DPI $)$ 

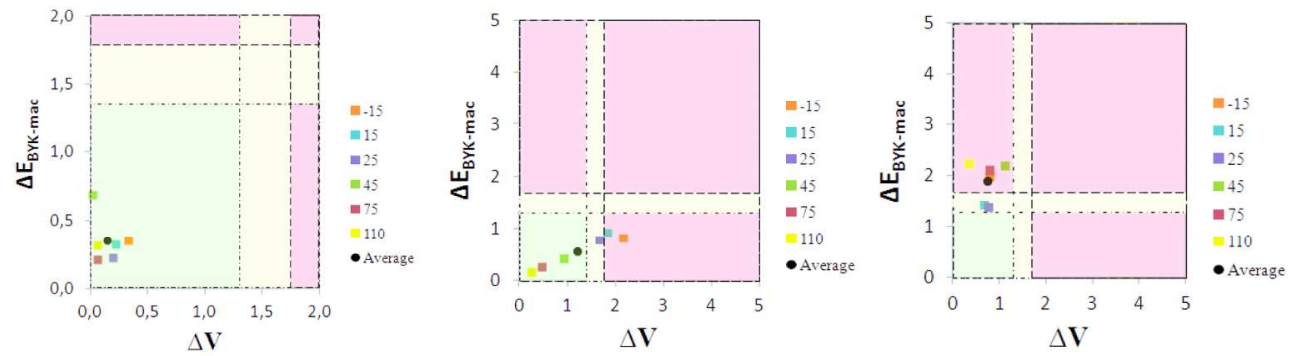

Figure 6: Color differences for all the measurement geometries for a) solid sample, b) pearlescent sample and c) metallic sample following the criterion Passes / Not Passed. The green region means almost indistinguishable color differences, the red zone means rejected color pairs and the yellow zone means critical color differences, samples need to be visually assessed by expert colorists. $177 \times 55 \mathrm{~mm}$ (300 x 300 DPI) 